\title{
Typical and Atypical Manifestations and Characteristics of the covid-19 Chest CT Scanner : Retrospective study of 28 patients
}

\author{
Jihane Habi *, Hind Guerroum *, Mariam Kassimi*, Nabil Chikhaoui *, Mohamed Mahi * \\ * Department of Radiology, Cheikh khalifa international university hospital, Mohammed VI university of health sciences. \\ DOI: 10.29322/IJSRP.10.07.2020.p10374 \\ http://dx.doi.org/10.29322/IJSRP.10.07.2020.p10374
}

\begin{abstract}
-
Objective:

The aim of our work was to describe the typical and atypical aspects in our retrospective study of 28 patients.
\end{abstract}

Methods: Scanning examinations were carried out on two scanners type 16 and 64 Bars with thin sections $(1.5 \mathrm{~mm})$. Data collection was done retrospectively from a reading made on the PACS system by two radiologists and helped by reports on the department's RIS system.

Results: The predominant typical damage found was a combination of central and peripheral bilateral ground glass opacity with consolidation associated with a high percentage of traction bronchiectasis and sub segmental vascular dilation. Atypical nodular, lymph node, and pleural injuries were very low in our series.

Conclusion: Atypical attacks are rare compared to typical known attacks. Typical disease may often be associated with crazy paving, bronchiectasis, and sub-segmental vascular dilations indicating signs of inflammatory disease severity.

\section{Keys points:}

1- Chest CT revealed mixed involvement (consolidation and ground glass opacity) predominant and crazy paving, with bilateral lung localization predominant in the lower right lobe.

2- Atypical damage (nodule, lymphadenopathy, and pleural damage) was very low at $4 \%$.

3- Bronchectasia was found at $32 \%$ and subsegmental vascular dilatation was found at $61 \%$, showing the seriousness of the lung involvement.

Index Terms- Lung- Radiology- Infection- Coronavirus

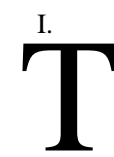
INTRODUCTION

The coronavirus was first declared in Wuhan on December 31, 2019 and declared as much as a global pandemic on March 11, 2020 by the World Health Organization (WHO). Its clinical presentation is similar to that of viral pneumonia and causes severe acute respiratory syndrome (SARS). The reference exam for its diagnosis is the real-time reverse transcription polymerase chain reaction (RT-PCR) method performed on samples from the respiratory tract. However, due to intrinsic limitations (i.e. collection and transportation of samples and diagnostic kit performance), sensitivity of RT-PCR at initial presentation ranges between $60 \%$ and $71 \%$ [1-2].

Because of the primary involvement of the respiratory system, chest CT is strongly recommended in suspected COVID-19 cases, for both initial evaluation and follow-up [3]. The CT sensitivity is $98 \%$ [4].

The aim of our study was to study the typical and atypical characteristics of CT lesions the 28 patients who have been diagnosed positive covid in our hospital and to illustrate the different manifestations found.

\section{REASERCH ELABORATION}

This study was approved by the Ethics Committee of our institution. Signed informed consent was obtained.

\section{Type of study:}

This is a retrospective study on the characterization of lesions on chest scanners of patients confirmed at Covid-19. The study spanned between April 1 and May 26, 2020.

\section{Data collection and analysis:}

Data collection was done retrospectively from a reading made on the PACS system by two radiologists and helped by reports on the department's RIS system.

It was based on the different morphological characteristics of the lesions (ground glass opacity, consolidation, mixed, crazy paving, reversed halo sign, pleural and lymph node involvement) and their lobar localizations. All scanners were produced without injection by a 16 and 64 bars scanner with thin sections $(1.5 \mathrm{~mm})$.

Hygiene and disinfection measures were established after each examination.

Exclusion criteria are scanners with breathing motion artifacts, normal scanners in Covid positive patients and scanners without positive PCR.

\section{Data analysis:}

Statistical analysis of all variables has been described through the use of descriptive statistics tools. Qualitative variables have been described in terms of percentage. The coding and the processing of the data were carried out on Excel Software. 


\section{RESULTS ANS FIDINGS}

Number of patients: 28

Average age: 57 ranging from 19 to 81

Patient gender: 19 men (67\%) / 9 women (32\%)

Imaging results:

The most common lesions are consolisolidation with ground glass opacity at $57 \%$, followed by ground glass opacity alone and crazy paving at $43 \%$. [Table I]

Bronchiectasis and sub-segmental vascular dilation were found at $32 \%$ and $61 \%$. Nodule, pleurisy and lymphadenopathy were found in few patients $(4 \%)$. [Table I]

Table I: The different characteristics with the number and percentage translation of patients reached.

\begin{tabular}{|l|l|l|}
\hline Lesion & $\begin{array}{l}\text { Number } \\
(\mathrm{n}=28)\end{array}$ & \multicolumn{1}{l|}{ Percentage } \\
\hline GGO (Groud glass opacity) & 12 & $43 \%$ \\
\hline Consolidation & 0 & $0 \%$ \\
\hline GGO+Conso & 16 & $57 \%$ \\
\hline Inetrlobular septal thickening & 6 & $21 \%$ \\
\hline Reticular pattern & 10 & $36 \%$ \\
\hline Crazy paving & 12 & $43 \%$ \\
\hline Nodule & 1 & $4 \%$ \\
\hline Bronchiectasis & 9 & $32 \%$ \\
\hline Pleural effusion & 1 & $4 \%$ \\
\hline Reversed halo sign & 1 & $4 \%$ \\
\hline Lymphadenopathy & 1 & $4 \%$ \\
\hline Sub-segmental Vascular dilatation & 17 & $61 \%$ \\
\hline
\end{tabular}

For the localization; we found bilateral lung localization predominant in the lower right lobe at $82 \%$. [Table II]

Table II: Number and percentage of lesion localization.

\begin{tabular}{|c|c|c|}
\hline Lesion localization & $\begin{array}{l}\text { Number } \\
(n=28)\end{array}$ & Percentage \\
\hline \multicolumn{3}{|l|}{$\begin{array}{l}\text { Centrale or peripheral } \\
\text { localization }\end{array}$} \\
\hline Peripheral zone & 12 & $43 \%$ \\
\hline Central zone & 0 & $0 \%$ \\
\hline Central + Peripheral zone & 16 & $57 \%$ \\
\hline \multicolumn{3}{|l|}{ Chest localization } \\
\hline Right & 6 & $21 \%$ \\
\hline Left & 1 & $4 \%$ \\
\hline $\mathrm{R}+\mathrm{L}$ & 21 & $68 \%$ \\
\hline \multicolumn{3}{|l|}{ Lobe Localization } \\
\hline Right upper lobe & 8 & $29 \%$ \\
\hline Right middle & 9 & $32 \%$ \\
\hline Right lower & 23 & $82 \%$ \\
\hline Left upper & 8 & $29 \%$ \\
\hline Left lower & 20 & $71 \%$ \\
\hline \multicolumn{3}{|l|}{ Number of lobe } \\
\hline 1 Lobe & 7 & $25 \%$ \\
\hline 2 Lobes & 9 & $32 \%$ \\
\hline
\end{tabular}

This publication is licensed under Creative Commons Attribution CC BY. http://dx.doi.org/10.29322/IJSRP.10.07.2020.p10374

\begin{tabular}{|l|l|l|}
3 Lobes & 6 & $21 \%$ \\
\hline 4 Lobes & 0 & $0 \%$ \\
\hline 5 Lobes & 5 & $18 \%$ \\
\hline
\end{tabular}

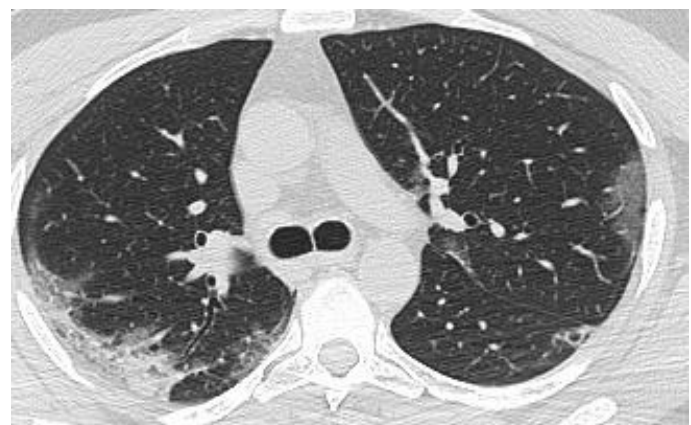

Figure 1: 46 year old presented cough with fever progressing for 10 days,

Axial CT showed ground glass opacity with condensation, bilateral peripheral with traction bronchiectasis.

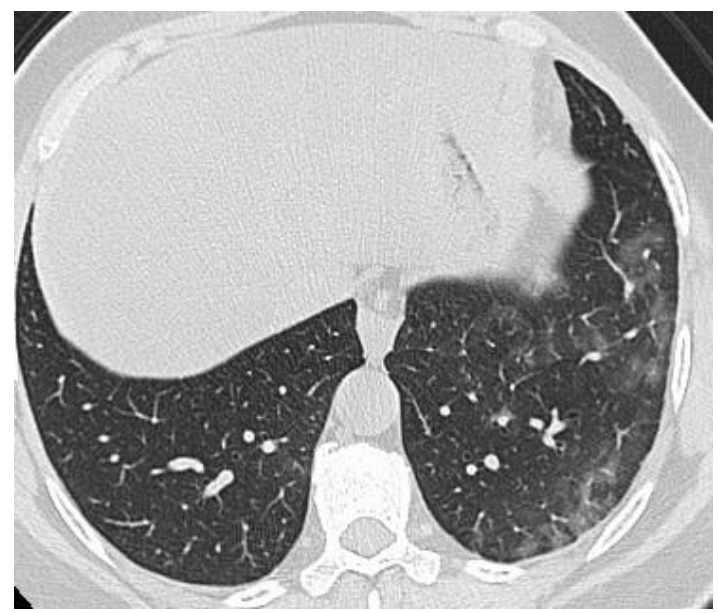

Figure 2: 62 years old, presented with fever and myalgia.

Axial CT scan showed; unilateral peripheral and central ground glass opacity.

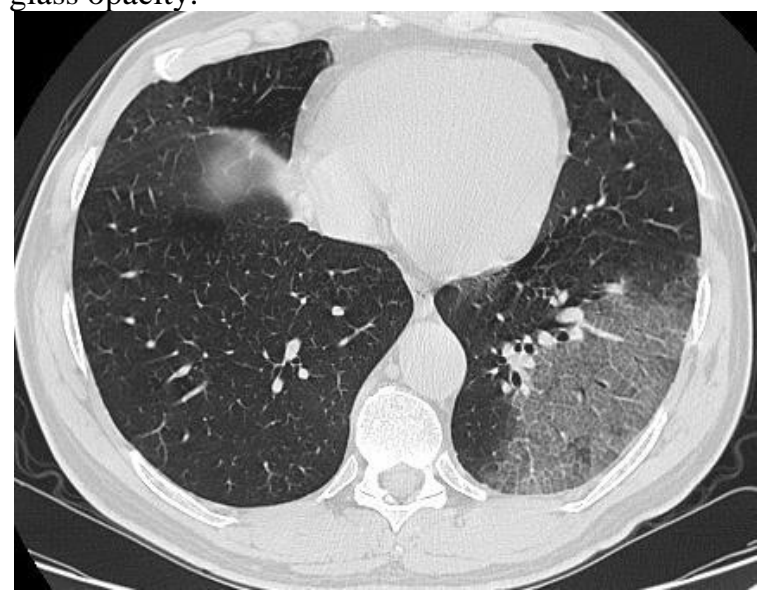

Figure 3: 62 years old, presented with fever, cough and abdominal pain.

Axial CT scan showed; unilateral Crazy paving with subsegmental vascular dilation. 


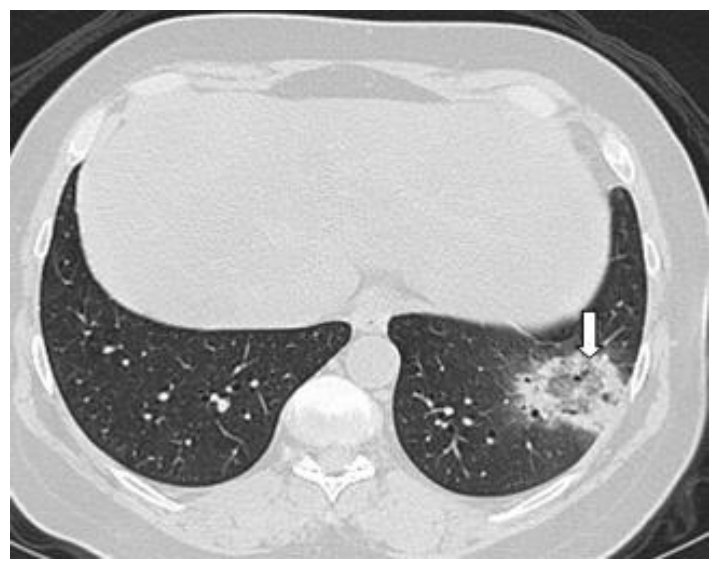

Figure 4: 60 years old, presented febrile cough with impaired general condition.

Axial CT scan showed reversed halo sign located on the lower left lobe (Arrow).

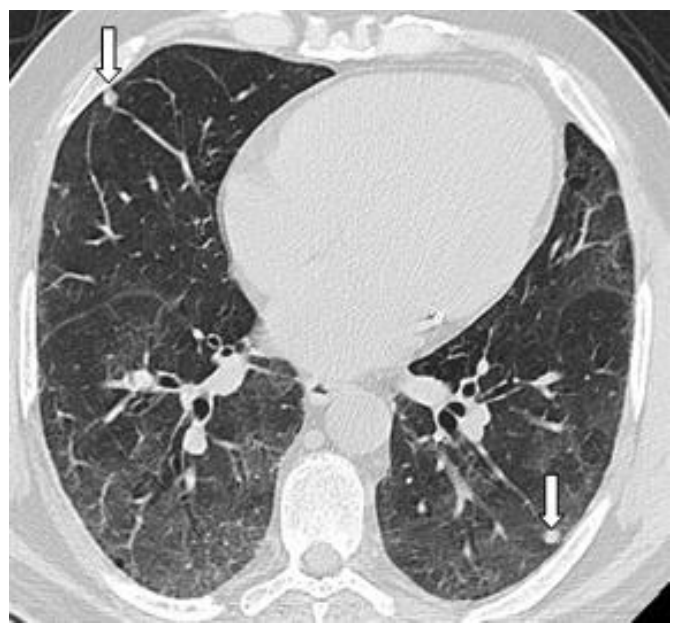

Figure 5: 50 years old, febrile dyspnea.

Axial CT scan showed ground glass opacity extended with two bilateral nodular lesions (Arrow).

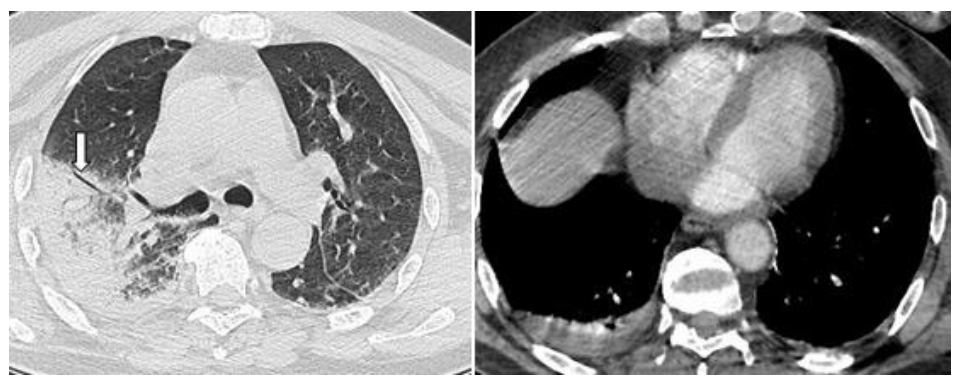

Figure 6: Patient is 65 years old, presented with severe respiratory distress with fever.

Axial CT scan showed an extensive right consolidation with traction bronchiectasis (Arrow) and homolateral pleurisy.

\section{RESULTS ANS FIDINGS}

Coronavirus is an infectious disease presented in the form of a global health crisis. Various studies on covid 19 imaging have been performed. In our establishment in Casablanca, Morocco, we conducted a study on the lesion characteristics of this pathology on the chest scanner of confirmed positive Covid patients. The most common typical scan injuries were peripheral ground glass opacity with consolidation associated with significant presence of Crazy paving, traction bronchiectasis and sub-segmental vascular dilatation attests to the seriousness of the pulmonary attack in our patients. The atypical scan aspect was nodule, lymphadenopathy, and pleurisy.

The average age of our patients was 57 compatible with that of the study by chung et al [5] with a predominant number of men at 19 $(67 \%)$ and a number of women at $9(32 \%)$ unlike the studies of Bernheim et al [6], Wu et al. [7] and Pan et al. [8] where the difference in gender is very small.

For the morphology of the various pulmonary lesions, our patient series revealed results slightly superior to the study by Bernheim et al [6] in ground glass opacity $43 \%$ vs $34 \%$. Mixed ground glass opacity and consolidation were found at a very high rate of $57 \%$ vs $41 \%$ found in Bernheim et al [6] and 29\% found in chung et al [5]. On the other hand, the presence of consolidation alone was not found in our series and differed from the two studies by Bernheim et al [6] at 2\% and Chung et al [5] at 29\%. We also noted that the presence of crazy paving is high by $43 \%$ vs $36 \%$ in li et al [9]; a percentage that exceeds the results of most studies. Nodule was described in one patient, $4 \%$ vs $13 \%$, which is the highest percentage found by the pan et al [10].

The presence of bronchiectasis at $32 \%$ far exceeds the studies of chung et al [5] and Bernheim et al [6] which not found this type of lesion. Pathogenesis can be the inflammatory damage of the bronchial wall and the bronchial obstruction, resulting in the destruction of bronchial wall structure, proliferation of fibrous tissue, fibrosis, and tractive bronchiectasis [11].

Sub-segmental vascular dilatation was significantly found in $61 \%$ compared to $89 \%$ in Damiano Caruso's study [12]. Ye et al suggested vascular enlargement may be due to pro- inflammatory factors [13].

Pleurisy and lymphadenopathies were found in one patient, which is very rare and consistent with the results of the literature found.

For the lesion site; our results are comparable to those of the study by $\mathrm{K}$. Wang et al. [14]; Most of the lesions were peripheral and central at $57 \%$ vs. $56 \%$ and $43 \%$ peripheral vs. $43 \%$, with no central involvement only.

Bilateral involvement of both lobes was predominant and was found to be $68 \%$ vs $76 \%$ in Chung et al [5]. Damage to the right lower lobe was found to be well over $82 \%$, which is in line with the results of the majority of the literature.

The main limitation of our study is the number of patients, which is barely 28 patients during one month of collection, thus allowing a study on a small sample. 


\section{CONCLUSION}

The atypical impairment are rare (nodule, lymphadepathy, and pleurisy), in contrast to the typical damage caused by the combination of peripheral and central basal ground glass opacity and consolidation. The high presence of crazy paving, traction bronchiectasis and sub-segmental vascular dilatation attests to advanced pulmonary inflammatory disease affecting patient management and prognosis.

\section{REFERENCES}

[1]: Yang Y, Yang M, Shen C et al. Evaluating the accuracy of different respiratory specimens in the laboratory diagnosis and monitoring the viral shedding of 2019-nCoV infections. 2020. doi: 10.1101/2020.02.11.20021493

[2] : Kanne JP, Little BP, Chung JH et al. Essentials for Radiologists on COVID19: An Update-Radiology Scientific Expert Panel. Radiology 2020:200527. doi: 10.1148/radiol.2020200527

[3] : Jin YH, Cai L, Cheng ZS, et al.; Zhongnan Hospital of Wuhan University Novel Coronavirus Management and Research Team; Evidence-Based Medicine Chapter of China International Exchange and Promotive Association for Medical and Health Care (CPAM). A rapid advice guideline for the diagnosis and treatment of 2019 novel coronavirus (2019-nCoV) infected pneumonia (standard version). Mil Med Res 2020; 7:4

[4] : Fang Y, Zhang H, Xie J et al (2020) Sensitivity of chest CT for COVID-19: comparison to RT-PCR. Radiology. https://doi.org/10. 1148/radiol.2020200432

[5] : Chung, MD et al. CT Imaging Features of 2019 Novel Coronavirus (2019-nCoV) Michael RSNA 2020-04-20

[6] : Bernheim A, Mei X, Huang M, Yang Y, Fayad ZA, Zhang N, et al. Chest ct findings in coronavirus disease-19 (COVID-19): relationship to duration of infection. Radiology. 0(0):200463.

[7] : Wu J, Wu X, Zeng W. Chest ct findings in patients with corona virus disease 2019 and its relationship with clinical features. Invest Radiol. 2020.

[8] : Pan Y, Guan H, Zhou S. Initial CT findings and temporal changes in patients with the novel coronavirus pneumonia (2019-nCoV): a study of 63 patients in Wuhan, China. Eur Radiol. 2020.

[9] : Kunhua Li JW, Wu F, Guo D, Chen L, Zheng F, Li C (2020) The clinical and chest CT features associated with severe and critical COVID-19 pneumonia. Invest Radiol. https://doi.org/10.1097/RLI. 0000000000000672

[10] : Pan F, Ye T, Sun P et al (2020) Time course of lung changes on chest CT during recovery from 2019 novel coronavirus (COVID19) pneumonia. Radiology. https://doi.org/10.1148/radiol. 2020200370

[11] : Hansell DM, Bankier AA, MacMahon H, McLoud TC, Muller NL, Remy J (2008) Fleischner Society: glossary of terms for thoracic imaging. Radiology 246:697-722

[12] : Damiano Caruso et al Chest CT Features of COVID-19 in Rome, Italy, April 2020.

[13] : Ye Z, Zhang Y, Wang Y et al. Chest CT manifestations of new coronavirus disease 2019 (COVID-19): a pictorial review. Eur Radiol 2020. doi: 10.1007/s00330-020-06801-0

[14] : K. Wang et al ; Imaging manifestations and diagnostic value of chest CT of coronavirus disease 2019 (COVID-

19) in the Xiaogan area. March 2020

\section{AUTHORS}

First Author - Jihane Habi, MD, habijihane@gmail.com. Second Author - Hind Guerroum, MD, guerroumhind@gmail.com and Mariam Kassimi,MD, M.Kassimi91@hotmail.com.

Third Author - Nabil Chikhaoui, PHD, nabil.chikhaoui@yahoo.fr and Mohamed Mahi, PHD, prmahimohamed@yahoo.fr.

Correspondence Author - Jihane Habi, habijihane@gmail.com ,+212662590217. 